\title{
Constraints on coda wave interferometry estimates of source separation: the acoustic case*
}

\author{
David Robinson ${ }^{1,2,4}$ Malcolm Sambridge ${ }^{1}$ Roel Snieder ${ }^{3}$ \\ ${ }^{1}$ Research School of Earth Sciences, The Australian National University, Canberra ACT 0200, Australia. \\ ${ }^{2}$ Risk Research Group, Geoscience Australia, GPO Box 378, Canberra 2601, Australia. \\ ${ }^{3}$ Center for Wave Phenomena and Department of Geophysics, Colorado School of Mines, \\ Golden CO 80401, USA. \\ ${ }^{4}$ Corresponding author. Email: david.robinson@anu.edu.au
}

\begin{abstract}
Synthetic experiments are used to test the applicability of coda wave interferometry (CWI) as a means for estimating distance between sources of nearby earthquakes. Acoustic waves for 45 sources are propagated through a Gaussian random medium. A pair-wise analysis of resulting waveforms illustrates the applicability of CWI as a tool for estimating source separation. Results suggest that, when the waveforms are filtered between 1 and $5 \mathrm{~Hz}$, CWI provides accurate estimates of the separation for source-pairs separated by $\delta<250 \mathrm{~m}$. The technique provides a lower bound on the actual separation when $\delta>250 \mathrm{~m}$. The CWI breakdown distance of $250 \mathrm{~m}$ is likely to vary with frequency content in the waveforms.

The interpretation of CWI source separation estimates is aided by the construction of a conditional probability density function (PDF) $P\left(\delta_{t} \mid \delta_{\mathrm{CWI}}\right)$, which describes the probability of actual separation $\delta_{t}$ for given CWI estimates $\delta_{\mathrm{CWI}}$. The conditional PDF provides a constraint on event separation that is asymmetric. It can be used independently of, or combined with, standard travel-time techniques to improve earthquake location.
\end{abstract}

Key words: earthquake location, coda waves, coda wave interferometry, relative location.

\section{Introduction}

Earthquake location is important for many applications. It indicates regions of relatively higher seismicity such as major lithospheric plate boundaries or local active source zones. Earthquake locations are required for magnitude determination, seismological studies of the Earth's interior and computing moment tensors. They are needed to understand strong motion and seismic attenuation and to model earthquake hazard or risk.

Uncertainty associated with absolute location techniques can be of the order of several kilometres. For example, Gutenberg and Richter (1939) considered events with location uncertainties of up to $2^{\circ}$ horizontally and $50 \mathrm{~km}$ vertically when studying the velocity of longitudinal waves at depth. More recently, Bondár et al. (2004) demonstrated that absolute locations are accurate to within $5 \mathrm{~km}$ with a $95 \%$ confidence level when local networks meet several station-related criteria. Such uncertainty is too large for studying micro-seismicity or re-constructing rupture surfaces from aftershock sequences.

Relative location techniques calculate displacements between pairs or groups of earthquakes. In principle, relative locations can be computed by differencing absolute locations. However, Pavlis (1992) shows that inadequate knowledge of velocity structure leads to systematic biases when relative positions are computed from absolute locations. This is confirmed by Richards et al. (2006), who state that inaccuracies in the travel-time velocity model are one of two primary error sources in absolute earthquake location, the other being noise in arrival-time picks. To reduce errors caused by the velocity model, relative location techniques constrain separation directly from travel-time differences between phases of two waveforms (Ito, 1985; Nadeau and McEvilly, 1997). By doing so, they remove errors associated with unknown lateral velocity variations outside the local region, because such variations influence all waveforms in a similar manner (Shearer, 1999). These techniques have achieved accuracies of the order of 15-75 $\mathrm{m}$ in local settings with good station coverage (Ito, 1985; Got et al., 1994; Waldhauser et al., 1999).

Precise relative location techniques have been applied to a diverse range of problems, such as the location of active fault planes (Deichmann and Garcia-Fernandez, 1992; Got et al., 1994; Waldhauser et al., 1999; Waldhauser and Ellsworth, 2002; Shearer et al., 2005), studying rupture mechanics (Rubin et al., 1999; Rubin, 2002b), interpreting magma movement in volcanoes (Frèmont and Malone, 1987), and monitoring pumping induced seismicity (Lees, 1998; Ake et al., 2005). Poupinet et al. (1984), Bokelmann and Harjes (2000), and Rubin (2002a) applied relative location techniques to identify earthquake doublets, events which occur at different times but have the same hypocentre and magnitude. Poupinet et al. (1984) use this information to measure temporal variation in crustal velocity, Bokelmann and Harjes (2000) use it to identify systematic temporal variations in seismic anisotropy, and Rubin (2002a) describes how it can be used to correct for timedependent station delays. 
All of these relative techniques are based on calculating travel-time differences between the first arriving parts of the waveform. That is, they typically use a cross correlation between two events to measure delay times between the arriving $\mathrm{P}$ and/or $\mathrm{S}$ waves. Information from the remainder of the waveform, including the coda, is discarded. The term coda refers to later arriving waves in the seismogram that arise from scattering (Snieder, 1999). Snieder and Vrijlandt (2005) demonstrate how coda wave interferometry (CWI) can be used to estimate source separation between earthquakes using the cross correlation of their coda waves. By using a different section of the waveform, the CWI technique has the potential to supplement other relative location techniques and improve locations further.

\section{Theory}

In CWI one uses variations in coda to constrain the relative location between two events. The main idea is that when the source position is perturbed, some ray paths are longer and some are shorter (Snieder, 2006). This notion is emphasised by defining the reference waveform, $\mathbf{u}(t)$ by

$$
\mathbf{u}(t)=\sum_{T} \mathbf{A}_{T}(t)
$$

and the perturbed waveform, $\tilde{\mathbf{u}}(t)$ resulting from the displaced source

$$
\tilde{\mathbf{u}}(t)=\sum_{T} \mathbf{A}_{T}\left(t-\tau_{T}\right),
$$

where $\tau_{T}$ is the travel-time perturbation on trajectory $T$ and $\mathbf{A}_{T}$ is the trajectory contribution to the waveform. The mean travel-time perturbation, $\mu_{\tau}$ obtained by integrating over all take-off angles from the source, vanishes when the scatterers are distributed homogeneously (Snieder and Vrijlandt, 2005). Variance of the travel-time perturbation $\sigma_{\tau}$ is not zero and can be related to the separation between the two sources. A summary of the theory follows.

Coda wave interferometry relies on a cross correlation between two waveforms. The cross correlation is computed for sliding windows across the entire length of the waveforms. The parameter of interest, the maximum cross correlation, is taken over all lag times in the sliding window. This differs from the relative location techniques which use a cross correlation to determine the lag time between early onset body-wave phases. Therefore, the value of the cross correlation is important in CWI but not in relative location techniques. The normalised cross correlation used in CWI is

$$
R^{\left(t, t_{w}\right)}\left(t_{s}\right)=\frac{\int_{t-t_{w}}^{t+t_{w}} u_{i}\left(t^{\prime}\right) \tilde{u}_{i}\left(t^{\prime}+t_{s}\right) d t^{\prime}}{\int_{t-t_{w}}^{t+t_{w}} u_{i}^{2}\left(t^{\prime}\right) d t^{\prime} \int_{t-t_{w}}^{t+t_{w}} \tilde{u}_{i}^{2}\left(t^{\prime}\right) d t^{\prime}},
$$

where $t_{s}$ is the shift time (or lag) and $t^{\prime}$ represents the integration variable. It is used to measure the change between the reference $u_{i}$ and perturbed $\tilde{u}_{i}$ displacement at the same recording station over a time window of length $2 t_{w}$ (Snieder and Vrijlandt, 2005). Note that $R^{\left(t, t_{w}\right)}(0)$ is the correlation coefficient and that the displacement $u$ can be replaced with other wavefields such as velocity or acceleration. Snieder (2006) demonstrates how the maximum of the normalised cross correlation in the time window is related to the variance of the travel-time perturbation $\sigma_{\tau}$ by

$$
R_{\max }^{\left(t, t_{t_{x}}\right)}\left(t_{s}\right)=1-\frac{1}{2} \bar{\omega}^{2} \sigma_{\tau}^{2}
$$

The square of the dominant angular frequency $\bar{\omega}^{2}$ is

$$
\bar{\omega}^{2}=\frac{\int_{t-t_{w}}^{t+t_{w}} \dot{u}_{i}^{2}\left(t^{\prime}\right) d t^{\prime}}{\int_{t-t_{w}}^{t+t_{w}} u_{i}^{2}\left(t^{\prime}\right) d t^{\prime}},
$$

where $\dot{u}_{i}$ represents the derivative of $u_{i}$ with respect to time, $t$. The relationship between the source separation $\delta$ and $\sigma_{\tau}$ is given by

$$
\delta^{2}=g(\alpha, \beta) \sigma_{\tau}^{2},
$$

where $\alpha$ and $\beta$ are $P$ and $S$ wave velocities, respectively. The function $g$ depends on the type of excitation (explosion, point force, double couple) and on the direction of source displacement relative to the point force or double couple. For example, for two double couple sources displaced in the fault plane,

$$
g(\alpha, \beta)=7 \frac{\left(\frac{2}{\alpha^{6}}+\frac{3}{\beta^{6}}\right)}{\left(\frac{6}{\alpha^{8}}+\frac{7}{\beta^{8}}\right)},
$$

whereas, for two point sources in a 2D acoustic velocity

$$
g(\alpha, \beta)=2 \alpha^{2}
$$

(Snieder and Vrijlandt, 2005). Note that equations (7) and (8) assume that sources have comparable source spectra. Practically, this can be achieved by filtering the waveforms so that the dominant frequency is less than the corner frequency of both events. When this criterion is satisfied the source behaves effectively like a point source in time, and the slip history is a step function (as seen by the waves with frequency less then the corner frequency). The importance of a change in orientation between the double couples when applying equation (7) is discussed by Robinson et al. (in press).

\section{Method}

In order to investigate the range over which CWI can be applied for estimating source separation, we present a numerical experiment with the following objectives:

1. Investigate the accuracy of CWI as a function of source separation. For example, how far apart can a pair of events be before the CWI breaks down?

2. Compute a conditional probability density function (PDF) to describe the likelihood of actual separation given a CWI estimate.

3. Explore the influence of the free surface, which is currently not considered in the theory, by repeating the experiments at three different depths.

To achieve these objectives we generate synthetic waveforms by solving the acoustic (or scalar) wave equation

$$
\frac{\partial^{2} \phi(x, z, t)}{\partial x^{2}}+\frac{\partial^{2} \phi(x, z, t)}{\partial z^{2}}=\frac{1}{\alpha^{2}} \frac{\partial^{2} \phi(x, z, t)}{\partial t^{2}}
$$

and apply the CWI theory. We solve equation (9) using a finite difference (FD) algorithm supplied by Heiner Igel (pers. comm., 2005, the code originally in MATLAB is re-coded in Fortran90 to improve efficiency). It is fourth order accurate in space and second order accurate in time. The model domain extends $80 \mathrm{~km}$ laterally and $40 \mathrm{~km}$ in depth and has reflective boundary conditions on all four boundaries (Figure 1). To avoid unwanted reflections from the boundaries we define a 'region of interest' with dimensions $20 \mathrm{~km}$ by $30 \mathrm{~km}$. Reflections from the sides and bottom of the model domain arising from events located within the region will not re-enter it in the time frame of 


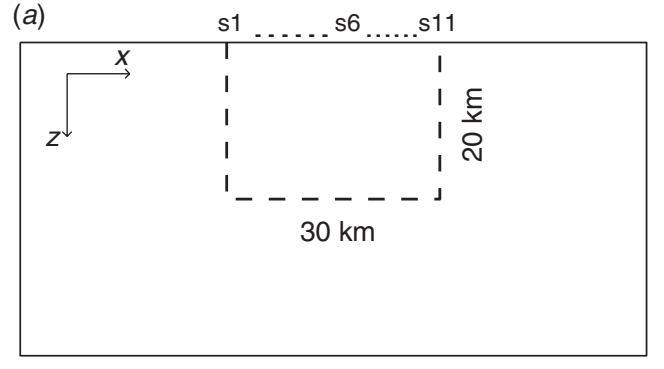

$80 \mathrm{~km}$ (b)

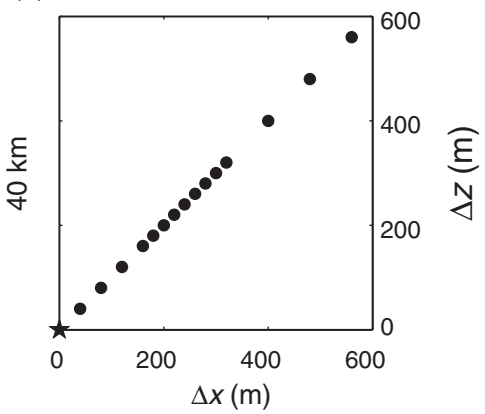

Fig. 1. (a) Model domain indicating reflective boundaries as solid lines. The 11 recording stations are equally spaced along the surface. They are referred to as s1, s2 to s11 from left to right. Reflections from the top surface represent the free-surface effect, whereas reflections from the bottom, left, and right boundaries for sources located within the dashed box ('region of interest') do not reach the stations within the time frame of the simulation. A small axes indicates the $x$ and $z$ orientation. (b) Spatial relationship between the reference (star) and perturbed (circles) sources.

the experiment. Reflections from the top of the model domain represent the free-surface effect. The grid separation in the medium is $20 \mathrm{~m}$.

A $P$ wave velocity model is created by computing a realisation of the Gaussian random media with mean velocity $\mu_{\alpha}=6 \mathrm{~km}^{-1}$, correlation length $a=1.2 \mathrm{~km}$ and standard deviation $\sigma_{\alpha}=1.5 \mathrm{~km}^{-1}$. Frankel and Clayton (1986) and Baig and Dahlen (2004) define Gaussian random media and provide techniques for creating them. Figure 2 illustrates the medium.

The scattering mean free path $l_{s}$ of a medium classifies the strength of scattering. It is the distance required for intensity to decay to $1 / e$ the value expected in the absence of scattering. Snieder and Vrijlandt (2005) demonstrate that CWI can be used to estimate source separation when

$$
l_{s}>>\lambda,
$$

where $\lambda$ represents the dominant wavelength of the scattered waves. Following a treatment similar to De Rosny and Roux (2001) and Scales and Van Wijk (2001), we define the intensity in a homogeneous medium by

$$
I_{h}(x)=\frac{I_{o}}{2 \pi x}
$$

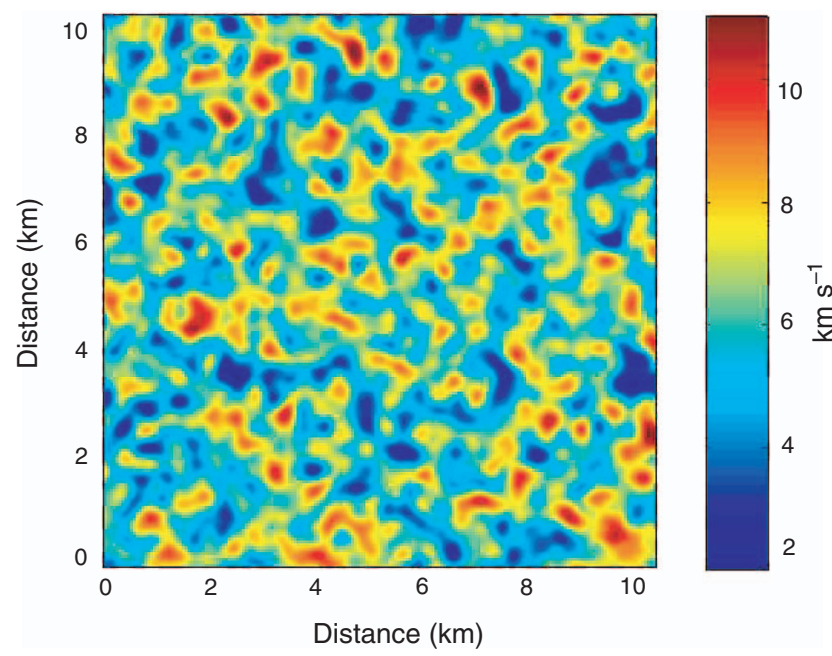

Fig. 2. $P$ wave velocity in a $10 \times 10 \mathrm{~km}$ section of the Gaussian random medium. where $x$ is the radial distance from the source, $1 / 2 \pi x$ is the geometrical spreading factor and $I_{o}$ is a source dependent intensity term. Note that equation (11) assumes no energy loss due to absorption. The intensity in the scattering medium is given by

$$
I_{c}(x)=\frac{I_{o}}{2 \pi x} e^{-x / l_{s}},
$$

where $e^{-x / l_{s}}$ describes the scattering-induced intensity decay. It follows that the scattering mean free path is related to the intensities by

$$
\frac{I_{c}(x)}{I_{h}(x)}=e^{-x / l_{s}} .
$$

A scatter plot of decay in intensity for heterogeneous, $I_{c}(x)$ versus homogeneous, $I_{h}(x)$ propagation is provided in Figure 3 for all grid points. Intensities $I_{c}(x)$ and $I_{h}(x)$ are approximated by $\max \left\{u^{2}(t)\right\}_{t}$. The yellow line represents the standard leastsquares fit to $\ln \left(I_{c}(x) / I_{h}(x)\right)$ versus $x$. It is influenced strongly by data at larger distances from the source at the expense of closer grid points. This leads to a poor fit at short distances. The problem occurs because the number of grid points located on the circumference of a source-centred circle grows with $r$ (i.e. there is more data at greater distances). The red line represents the best fit when a weighted least-squares algorithm is chosen with data weights defined by $1 / r$. This approach results in an improved fit at shorter distances. The slope of the red line is used to define the mean free path $l_{s}$ in the medium (i.e. from equation (13)). The mean free path is $7.3 \mathrm{~km}$. Note that intensity and hence $l_{s}$ depend on frequency content of the waveforms and will therefore vary with filtering.

Three reference events are defined at different depths in the model domain. The event depths are denoted shallow, mid, and deep corresponding to increasing depths with respect to the mean free path. The shallow reference source is buried at a depth of $0.4 l_{s}$ (or $\left.3 \mathrm{~km}\right)$, the mid source $0.7 l_{s}$ (or $5 \mathrm{~km}$ ) and the deep source $2.1 l_{s}$ (or $15 \mathrm{~km}$ ). Applicability of the CWI technique is tested by considering perturbations from each of the three reference sources.

All sources are modelled as line sources perpendicular to the model domain with source time functions defined by a Ricker wavelet of form

$$
s(t)=\left(1-2 \pi^{2} f_{o}^{2}\left(t-t_{o}\right)^{2}\right) e^{-\pi^{2} f_{o}^{2}\left(t-t_{o}\right)^{2}},
$$




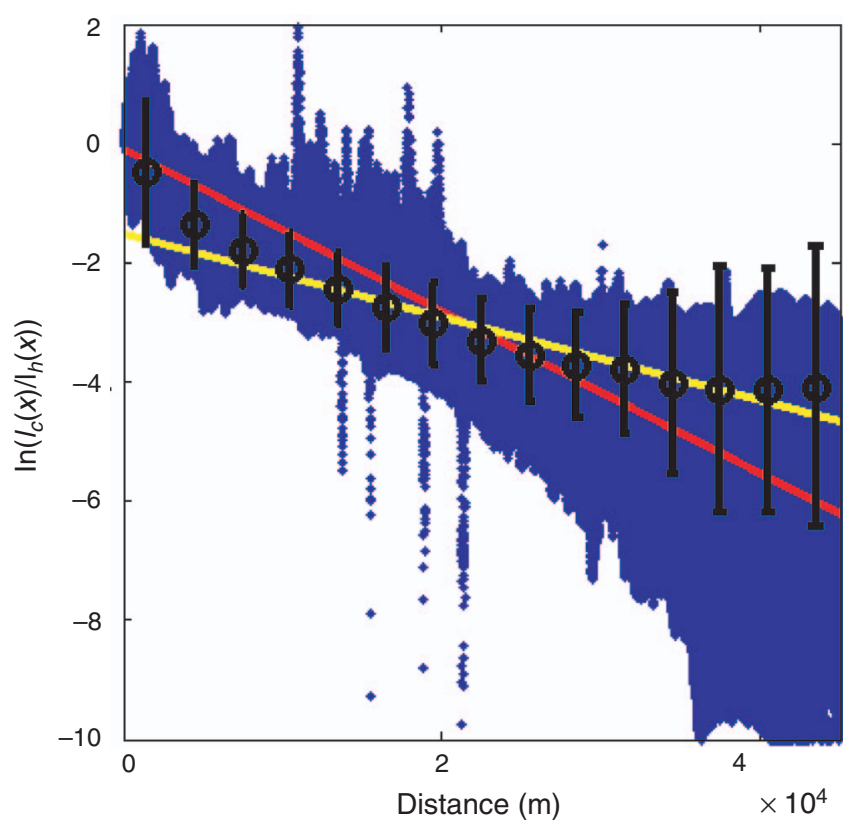

Fig. 3. Scatter plot showing the logarithm of relative decay in waveform intensity between homogeneous and heterogeneous propagation ( $y$-axis) versus the distance in metres from the source location (x-axis). The intensities $I_{c}(x)$ and $I_{h}(x)$ represent the intensity in the heterogeneous and homogeneous velocity models, respectively. Error bars indicate the mean and standard deviation (tails) of intensity ratio $\ln \left(I_{c}(x) / I_{h}(x)\right)$ when the data is sorted into distance bins of width $3 \mathrm{~km}$. The yellow line represents a standard least-squares fit and the red one a least-squares fit with data weights defined by 1 over the radial distance from source. The mean free path $l_{s}$ of the medium is computed from the red line's slope. That is, we favour the data at short distance when computing $l_{s}$. Data at greater distances are more likely to be effected by artificial reflections from the boundaries of the model domain.

with dominant frequency, $f_{0}=8 \mathrm{~Hz}$ and time delay, $t_{0}=0 \mathrm{~s}$. The time step used in the finite difference calculation is defined by

$$
\Delta t=\frac{\Delta x}{2 \alpha_{\max }},
$$

where $\Delta x=20 \mathrm{~m}$ is the grid separation and $\alpha_{\max }$ is the maximum velocity in the medium. Note that equation (15) satisfies the Courant, Friedrichs, Lewy (CFL) condition (Courant et al., 1928). This ensures that the propagating wave cannot travel more than one half of any cell within a single time step, which is a necessary condition for the stability of the simulation. Reference waveforms are computed at stations S1 to S11 (see Figure 1) for each of the 3 reference events.

Perturbed sources are defined at progressively increasing distances from each reference source in a diagonal direction towards the upper right corner of the model domain (Figure 1). They are defined at equi-distant grid separations (from the reference source) in the positive $x$ - and negative $z$ - directions of $2,4,6,8,9,10,11,12,13,14,15,16,20,24$, and 28 grid points, corresponding to actual separations of 57, 113, 170, 226, $255,283,311,339,368,396,424,453,566,679$, and $792 \mathrm{~m}$, respectively. The finite difference software is used to generate waveforms at stations S1 to S11 for all perturbed sources resulting in 11 waveforms for each of the 15 perturbations from the 3 reference sources (i.e. a total of 495 perturbed waveforms). Note that the same source time function is used for all simulations.

\section{Results}

Coda wave interferometry source separation theory is applied to sliding windows of length $0.75 \mathrm{~s}$ for each of the referenceperturbed pairs on a station-by-station basis for all pairs sharing the same reference depth. This analysis results in roughly 14 station specific estimates of separation (a total of 154 estimates of separation across all stations) for a given perturbation - source depth.

The acoustic wave propagation is illustrated in Figure 4 with six snapshots in the 'region of interest' for the mid depth reference source. Recall that the mid depth corresponds to roughly $0.7 l_{s}$ and represents an actual depth of $5 \mathrm{~km}$. The direct wave (outermost arc) and the reflection from the free surface (innermost arc) are clearly depicted. Strong scattering is observed by the colour variation following each of the major arcs. Coda wave interferometry theory does not explicitly consider reflections from the free surface. The decision to repeat numerical experiments at three depths is motivated by the strength of the surface reflection in Figure 4 and the unknown influence that it could have on CWI separation estimates.

Recording station S6 is represented by the triangle in Figure 4 and the associated waveform is shown in the top panel of Figure $5 a$ in blue. The red waveform is computed at the same station when the event is perturbed by $\Delta x=8$ and $\Delta z=8$ grid cells (i.e. roughly $226 \mathrm{~m}$ ) in the manner described in Figure 1. The second panel of Figure $5 a$ emphasises the similarity of the first arrivals (left) and differences between the coda (right) for the reference and perturbed sources. The high level of similarity between direct arrivals would not be observed if the source properties varied significantly.

The third panel illustrates the maximum cross correlation and the fourth panel the actual (blue dashed) and CWI computed (green solid) separation. Note that the waveforms are filtered between 1 and $5 \mathrm{~Hz}$ using a Butterworth filter before calculating the maximum cross correlation and source separation.

Figure $5 b$ illustrates the corresponding data when the reference and perturbed sources are moved to the deep location. This depth corresponds to roughly $2.1 l_{s}$ (equivalent to $15 \mathrm{~km}$ ). As with the mid depth, the figure illustrates similar direct arrivals and different coda. The third panels of Figures $5 a$ and $b$ illustrate a high level of maximum cross correlation for early windows, which is associated with the similarity of the first arrivals. This high correlation is followed by a decay as the sliding windows move further into the coda.

For the middle source depth the maximum cross correlation flattens and fluctuates between $0.7 \pm 0.1$ for sliding window centroids exceeding $2 \mathrm{~s}$. The CWI separation estimates for the mid depth are shown in the fourth panel of Figure $5 a$. These start at zero and increase until sliding window centroid $2 \mathrm{~s}$, after which they fluctuate around the actual separation of $226 \mathrm{~m}$.

The underestimation of separation for initial sliding windows occurs because the waves reaching the receiver at early times result from scattered waves that have not propagated in all directions from the source (Snieder and Vrijlandt, 2005). That is, more time is required for those waves that began their propagation away from the receiver to be scattered back towards it. Snieder (2004) explains how fluctuations in the separation estimates can be related to cross terms that are ignored in the CWI theory. The influence of these cross terms decreases with increasing sliding window size. However, a larger window size leads to fewer independent estimates of separation. A preliminary investigation revealed that a window length of $0.75 \mathrm{~s}$ provides a healthy balance between fluctuation and the number of independent separation estimates for these synthetic waveforms. 

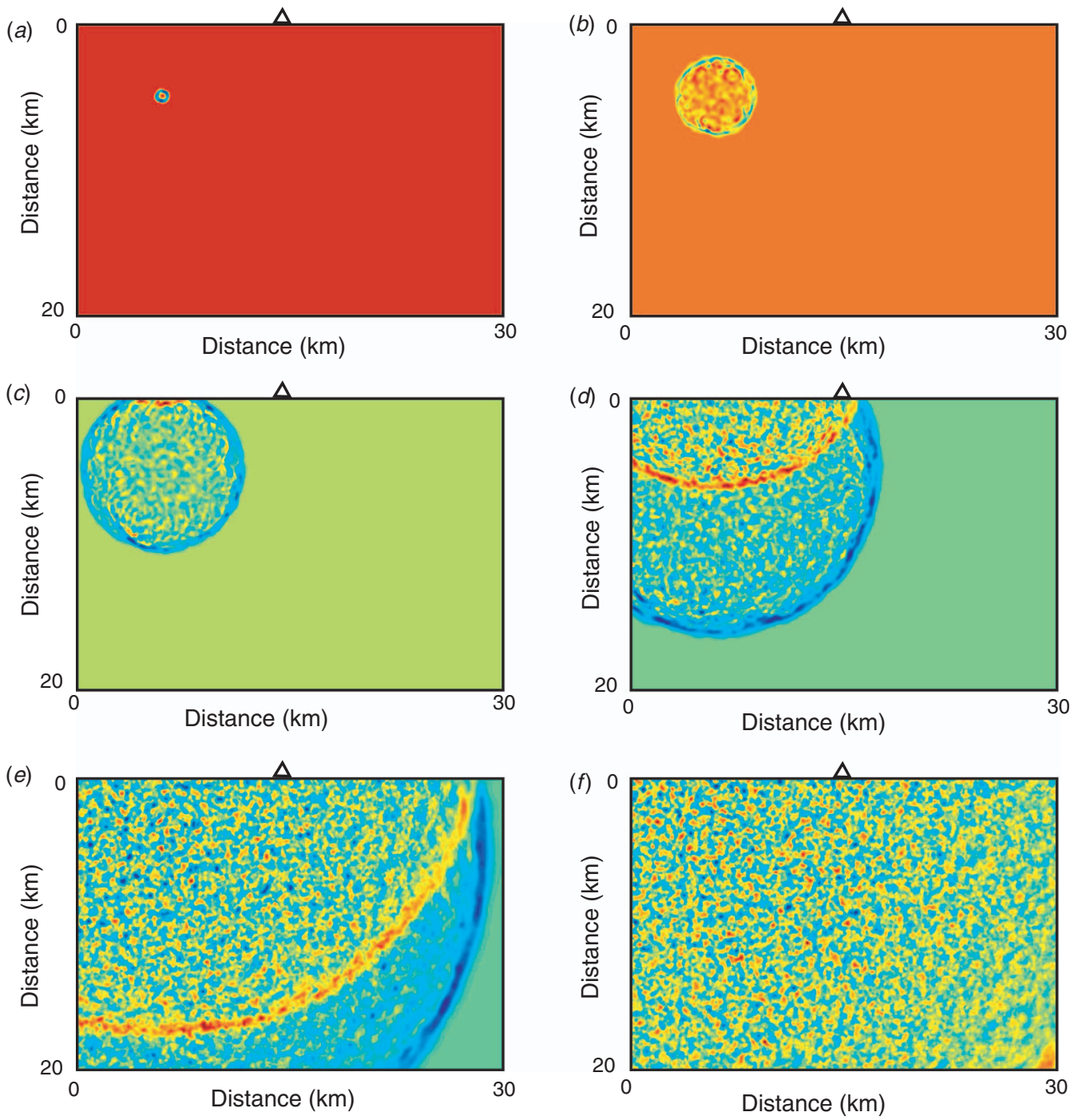

Fig. 4. Snapshots of the modelled scalar potential $\phi$ in the region of interest for the mid reference event at times $(a) 0.1$, (b) $0.5,(c) 1,(d) 2,(e) 4$, and $(f) 6 \mathrm{~s}$. The S6 receiver location is illustrated by the triangle. Note that the colour map is re-scaled in each snapshot to assist visualisation of $\phi$ throughout the entire model domain. The corresponding $\phi$ at receiver location S6 is shown as a function of time in blue in Figure $5 a$.

For the deep source (Figure $5 b$ ), the maximum cross correlation decays until $5.8 \mathrm{~s}$ and then increases again. The separation estimates (panel 4) start low, rise to the actual separation and then decrease for sliding windows where the maximum cross correlation is increasing. The cause of this turning point is not clear. Preliminary investigations have suggested that it is neither related to artificial boundary reflections nor local anomalies. In summary, the CWI performs marginally worse for deeper sources than it does for the mid one.

Figure 6 illustrates separation estimates as a function of running window centroid for six of the perturbations. The estimates are illustrated in subplots Figure $6 a-f$ for perturbations of $57,170,226,255,311$, and $424 \mathrm{~m}$, respectively. The three panels in each subplot represent the shallow (top), mid (middle) and deep (bottom) sources. The estimated separation from each of the 11 stations is plotted as a thin grey line, the mean separation across all stations in thick green and the actual perturbation in dashed blue.

Subplot $6 a$ illustrates a clear scatter in station specific estimates. The mean separation estimate for the shallow and deep depth configurations are accurate, but the separation estimates for the mid reference depth is biased above the actual separation. Preliminary studies with other realisations from the same Gaussian random medium have indicated that this bias is related to a local anomaly.

The format of Figure $6 a$ is repeated for increasing perturbations from $170 \mathrm{~m}$ in Figure $6 b$ to $424 \mathrm{~m}$ in Figure $6 f$. The scatter of the grey station specific estimates is evident for all perturbations and source depths. The mean estimates for the shallow sources are close to the actual separation for all perturbations less than $226 \mathrm{~m}$. They are less than the actual separations for perturbations of $255 \mathrm{~m}$ and higher. For perturbations between 170 and $255 \mathrm{~m}$ the CWI separation estimates perform well for the mid depth, but thereafter the technique under-estimates the actual separation. For the deep sources there is a turning point in the separation estimate for actual perturbations greater than 57 but less than $311 \mathrm{~m}$. Generally, the deep CWI values under-estimate the actual separation.

On average the estimates for the deep sources are marginally lower than the actual separation. Estimates for the shallow and mid sources are accurate for actual separations less than $255 \mathrm{~m}$. However, there is a small upper bias in the mid depth 
(a)
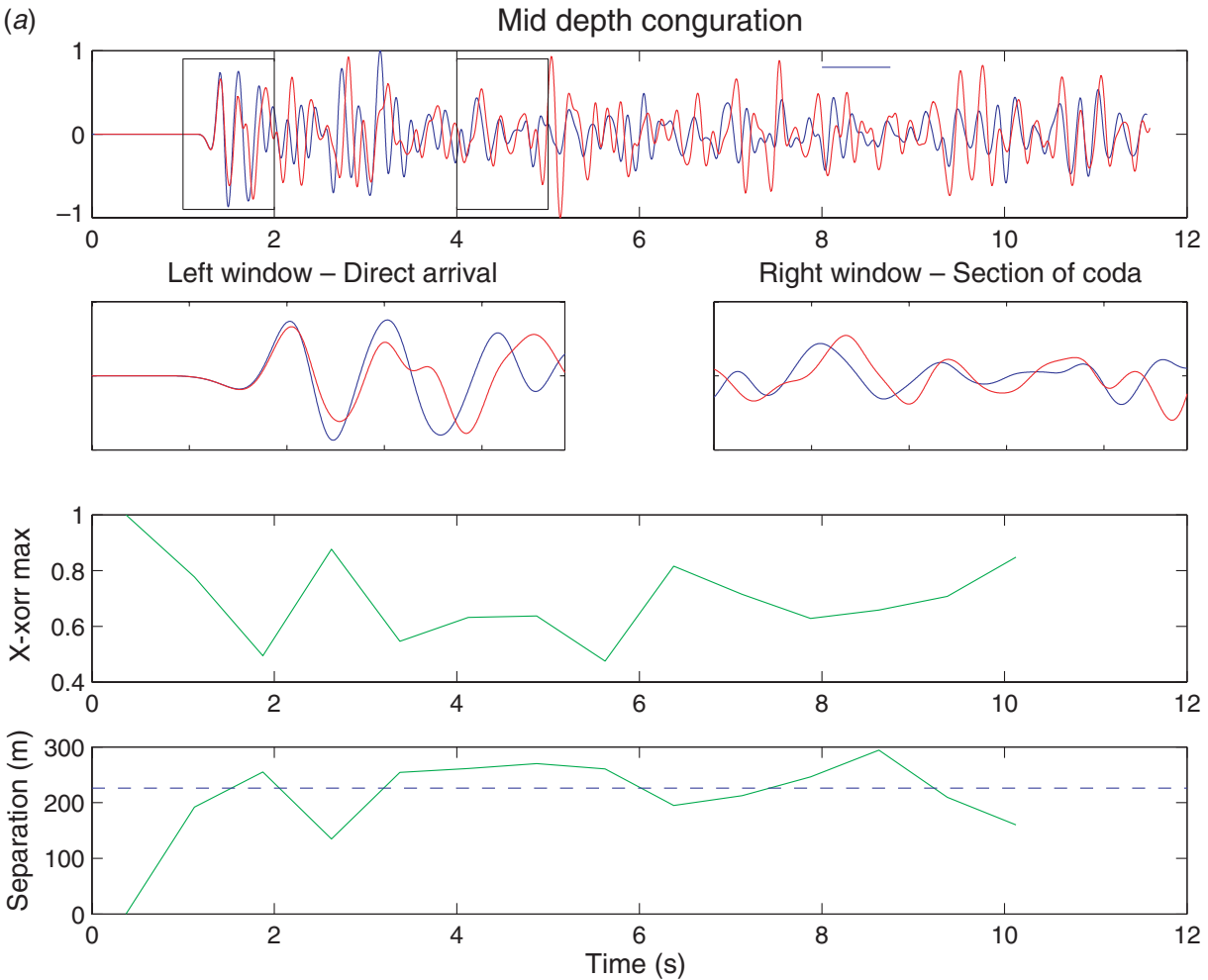

(b)
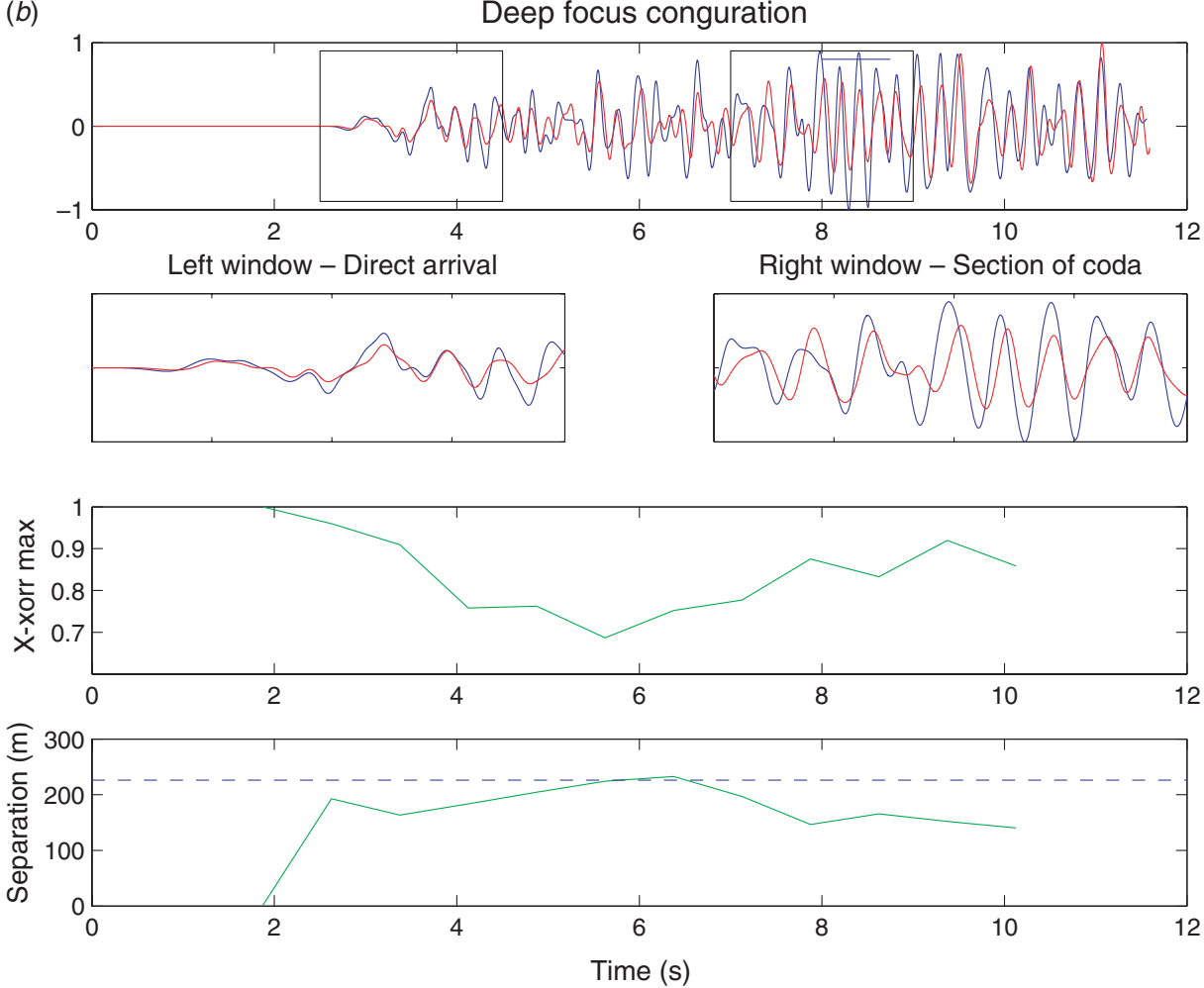

Fig. 5. CWI estimates in medium 1 for a perturbation of $226 \mathrm{~m}$ at (a) mid and (b) deep source depths. In each case the top panel illustrates the reference (blue) and perturbed (red) waveforms $(\phi)$ at S6 after normalisation to amplitude 1 . The blue horizontal line represents the sliding window length used to compute the cross correlations. The left box surrounding the direct arrival and the right box surrounding a section of coda are shown in more detail in the second panel. The third panel displays the maximum cross correlation as a function of sliding window centroid and the fourth panel illustrates the estimated separation (green) and actual separation (blue)

configuration for actual separations less than $170 \mathrm{~m}$. There is no evidence in this experiment to suggest that the free surface has a detrimental effect on CWI source separation estimates. Rather, the poorer performance of CWI for determining separations at the deep focus suggests that there may be limits to the acceptable source-to-station distance. We return to this point later. 
(a)
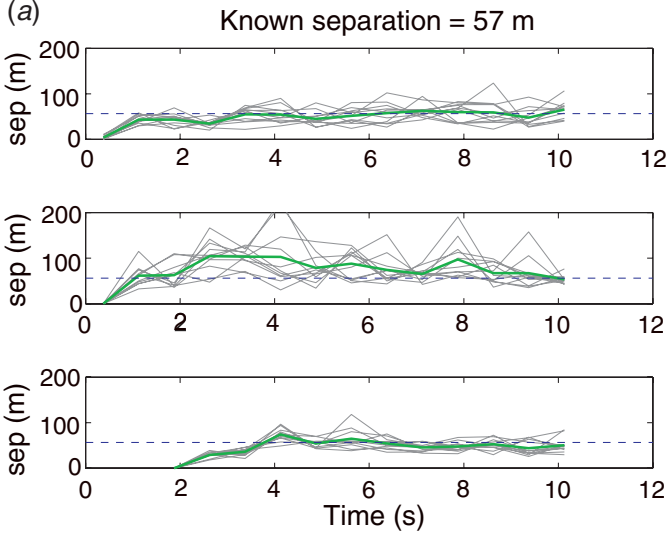

(c)
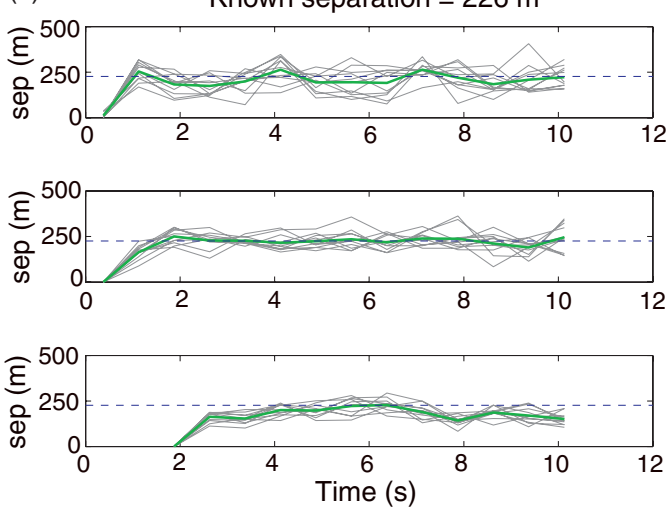

(e)
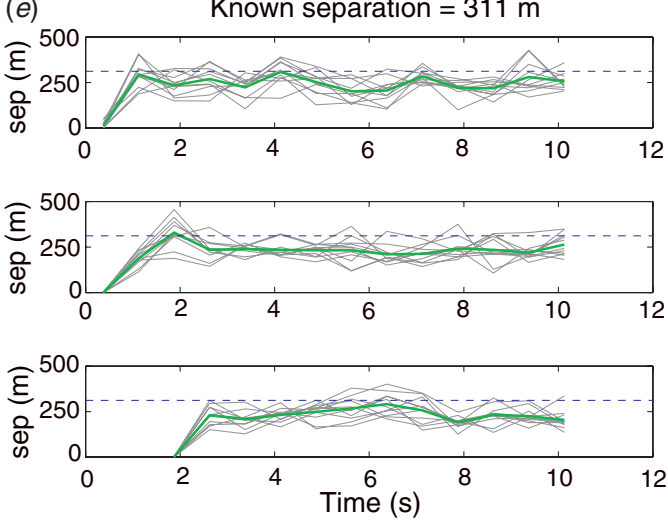

(b)
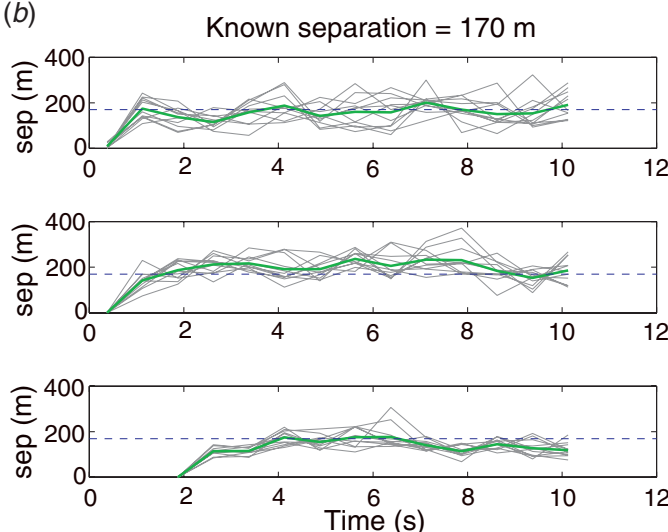

(d)
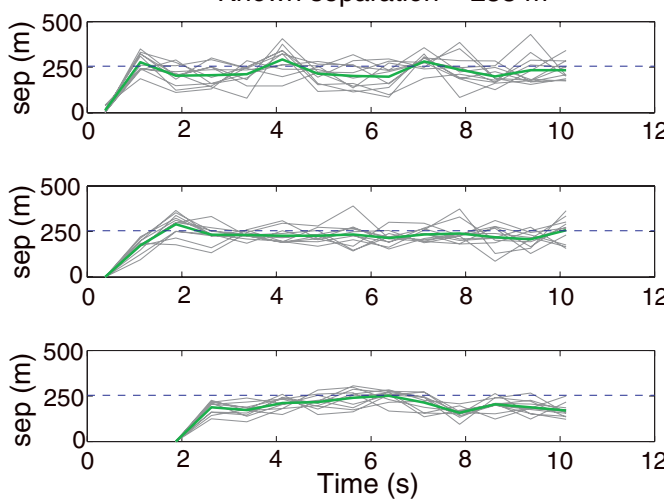

$(f)$
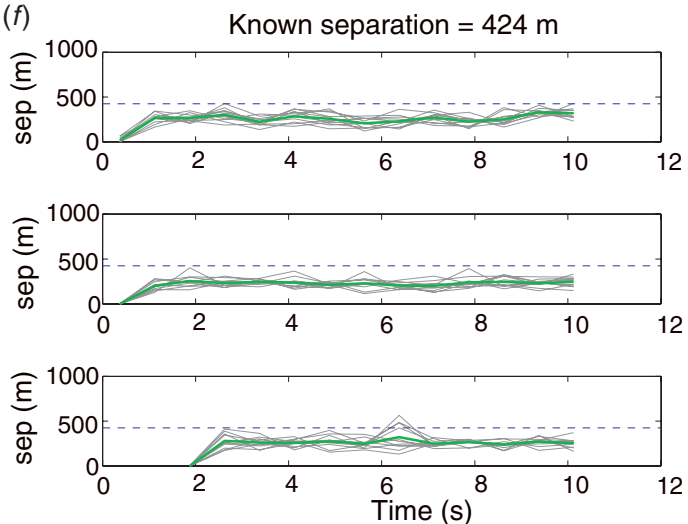

Fig. 6. Separation estimates for medium 1 as a function of sliding window. The 11 thin coloured lines represent CWI estimates from the 11 synthetic stations, the thick green line indicates the mean from all stations, and the dashed blue line the true separation. Three rows are shown in each subfigure to represents the shallow (top), mid (middle) and deep (bottom) source experiments. Note that the $y$-scale is consistent for each row of a subfigure but varies between subfigures.

Separation estimate error bars are illustrated in Figure 7 for all perturbation depth combinations. The error bars confirm that CWI separation estimates are accurate for perturbations up to $\sim 250 \mathrm{~m}$. When the actual separation exceeds $250 \mathrm{~m}$ the CWI estimates continue to fluctuate around $250 \mathrm{~m}$. Such error bar plots assist us by illustrating when CWI estimates of source separation breakdown. However, they offer little practical benefit in interpreting CWI estimates from real observations because the actual separation is rarely known. For this purpose it is convenient to represent the results of the synthetic experiments with a conditional probability density function, $P\left(\delta_{t} \mid \delta_{\mathrm{CWI}}\right)$ which describes the probability density of actual separation $\delta_{t}$ given the CWI separation estimate $\delta_{\mathrm{CWI}}$.
The conditional PDF $P\left(\delta_{t} \mid \delta_{\mathrm{CWI}}\right)$ is obtained by the following procedure:

1. Combining estimates for all three depths to give a single error bar for each perturbation (Figure 8)

2. Selecting a CWI estimate of interest (e.g. the red dashed line at $\delta_{\mathrm{CWI}}=200 \mathrm{~m}$ in Figure 8).

3 . Evaluating the probability density at each of the actual separations by assuming that its associated error bar defines a normal distribution for $P\left(\delta_{\mathrm{CWI}} \mid \delta_{t}\right)$. This assumption is supported by the histograms of $\delta_{\mathrm{CWI}}$ at $\delta_{t}=226$ and $311 \mathrm{~m}$ in Figure 8.

4. Fitting a smooth curve through the probability density estimates obtained in Step 3 using cubic splines and re- 
normalising the data so that the area under the curve integrates to 1 .

5. Repeating Steps 1 to 4 for all $\delta_{\mathrm{CWI}}$ of interest.

The black, blue and red lines in Figure 9 illustrate $P\left(\delta_{t} \mid \delta_{\mathrm{CWI}}\right)$ for $\delta_{\mathrm{CWI}}$ of $50,100,150,200,250,300,350$, and $400 \mathrm{~m}$ when all stations, the single station S1 and stations S1 to S5 are considered, respectively. Probability density peaks are observed in each conditional PDF around the CWI estimate for $\delta_{\mathrm{CWI}}$ of $50,100,150$, and $200 \mathrm{~m}$. This implies that the CWI separation estimate is more likely to be around the actual separation than not. For $\delta_{\mathrm{CWI}}=250 \mathrm{~m}$ the peak when all stations are considered is only just noticeable and for estimated separations greater than $300 \mathrm{~m}$ the peak occurs at separations exceeding the estimate. Contrastingly, the peak remains more prominent when the single station S1 or stations S1 to S5 are considered. This observation provides further support for the suggestion that there is a limit to the source-to-station distance because stations S6 to S11 are further from the source than station $\mathrm{S} 1$ to $\mathrm{S} 5$.

It is worth reflecting on the primary difference between Figures 8 and 9. Figure 8 demonstrates that CWI estimates are accurate for actual separations up to $250 \mathrm{~m}$. However, Figure 9 demonstrates that if the CWI estimate is $250 \mathrm{~m}$ there is roughly equal probability that the actual separation is any where between 250 and $800 \mathrm{~m}$. The situation worsens for $\delta_{\mathrm{CWI}}=400 \mathrm{~m}$ where the probability density suggests that the actual separation is more likely to be $500 \mathrm{~m}$. This observation, hereafter termed the rising phenomena, should be interpreted with care because calculation of the conditional PDF for larger $\delta_{\mathrm{CWI}}$ is dominated

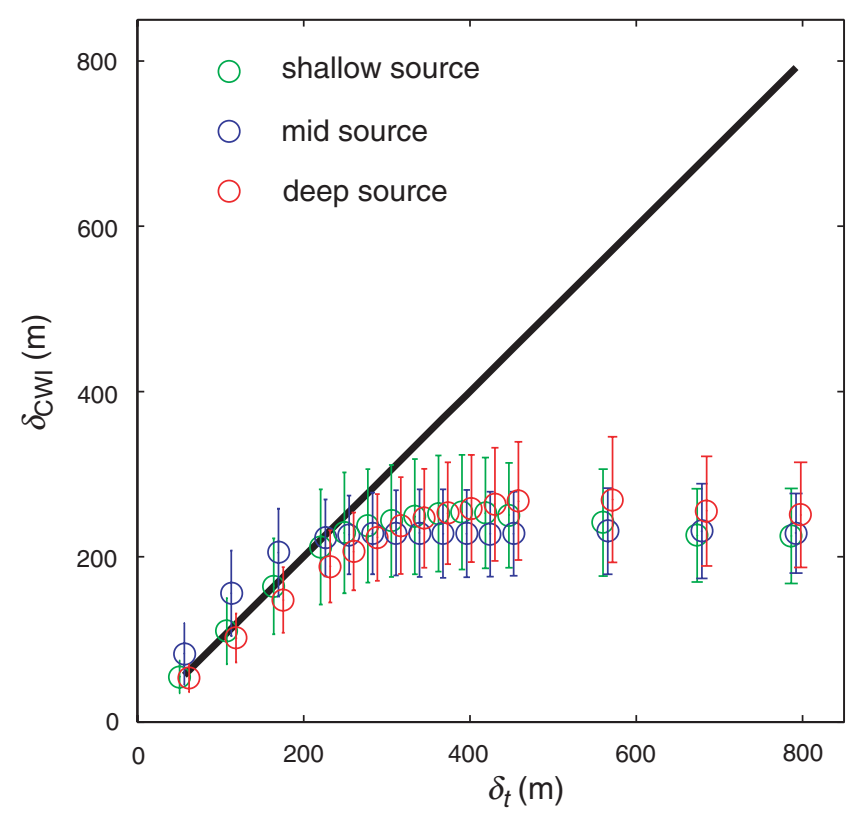

Fig. 7. Separation estimates after Butterworth filtering between 1 and $5 \mathrm{~Hz}$. An individual error bar represents the mean (central circle) \pm 1 standard deviation (tails). For each perturbation-depth combination there are 11 stations with 14 sliding windows, amounting to 154 separation estimates. The first five computed separations for each station are removed to ensure that initial estimates, demonstrated to under-estimate the actual separation, are ignored. That is, a total of 99 estimates are used to compute each error bar. The error bars are plotted as a function of increasing perturbation (or actual separation) and are shown in green, blue, and red for shallow, mid and deep sources, respectively. The shallow (green) and deep (red) error bars have been translated to the left and right, respectively to assist visualisation. by small probability densities computed from tails of the normal distributions (i.e. error bars in Figure 8).

Figure 10 represents the 2D conditional PDF obtained by repeating Steps 1 to 4 for CWI estimates at increments of $2 \mathrm{~m}$ and re-normalising all density estimates so that the volume underneath the surface integrates to 1 . Concentration of probability density around the diagonal line occurs when the CWI estimate is close to the actual separation. For example, concentration in the lower left corner confirms the accuracy of CWI at short distances. A second concentration located to the right of the diagonal for $\delta_{\mathrm{CWI}}>300 \mathrm{~m}$ is related to the rising phenomena described above. Even when the CWI estimates are $400 \mathrm{~m}$ the $2 \mathrm{D}$ conditional PDF tells us something about the probability density of different actual separations.

An alternative view of the 2D PDF is given by Figure 11, which illustrates probability that the CWI estimate is within \pm 30 m of the actual separation. For low CWI estimates of around $60 \mathrm{~m}$ there is a probability of 0.5 that the estimate is within $\pm 30 \mathrm{~m}$ of the actual separation. For estimated separations of 100,150 , and $200 \mathrm{~m}$ there are probabilities of $0.39,0.18$, and 0.11 that the estimated separations are within $\pm 30 \mathrm{~m}$ of the actual separations, respectively. Figure 11 suggests that the best result over the broadest range of event separations is achieved when stations S1 to S5 are considered.

\section{Discussion}

We use synthetic experiments to explore the range of applicability of CWI for determining the separation between pairs of earthquakes. A finite difference solver is used to model acoustic wave propagation in a $2 \mathrm{D}$ velocity model for line sources at increasing separations from one another. We have shown that CWI is most sensitive in the range $10-200 \mathrm{~m}$ and that accurate estimates of separation can be attained from a single station. This has the potential to provide further constraint on relative locations that are independent of arrival-time data. We anticipate

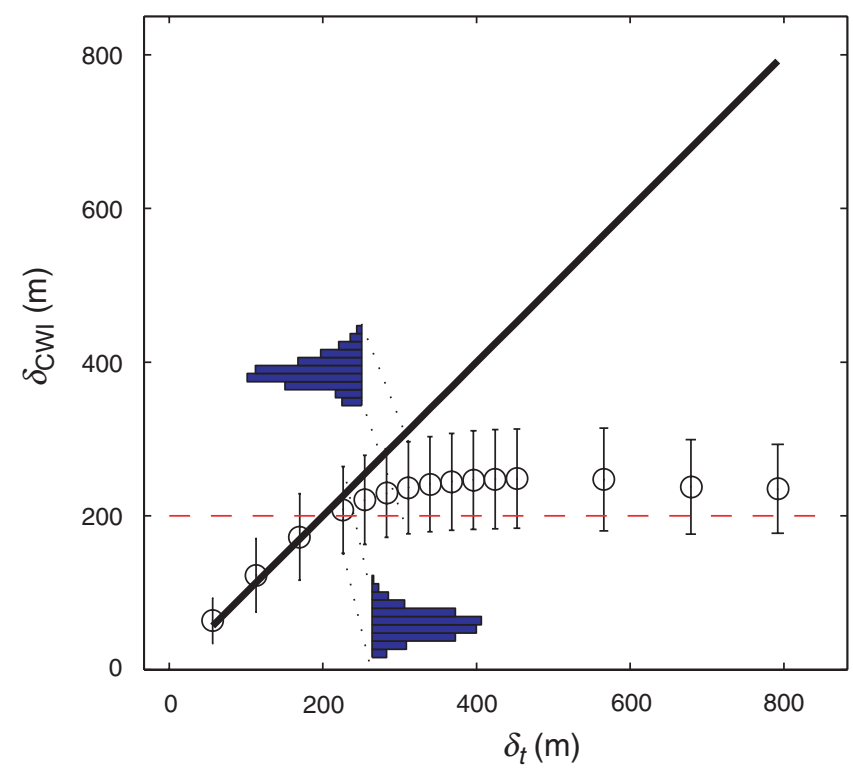

Fig. 8. Separation estimates when data from all depths are aggregated. The error bars follow the same format as those described in Figure 7. The dashed red line demonstrates the choice of a fixed $\delta_{\mathrm{CWI}}$ in step 2 of the process to compute $P\left(\delta_{t} \mid \delta_{\mathrm{CWI}}\right)$. Histograms illustrate that the distribution of $\delta_{\mathrm{CWI}}$ is normal at $\delta_{t}=226 \mathrm{~m}$ (bottom) and $\delta_{t}=311 \mathrm{~m}$ (top), a requirement at step 3 . 
(a) $\quad P\left(\delta_{t} / \delta_{\mathrm{CWl}}=50 \mathrm{~m}\right)$

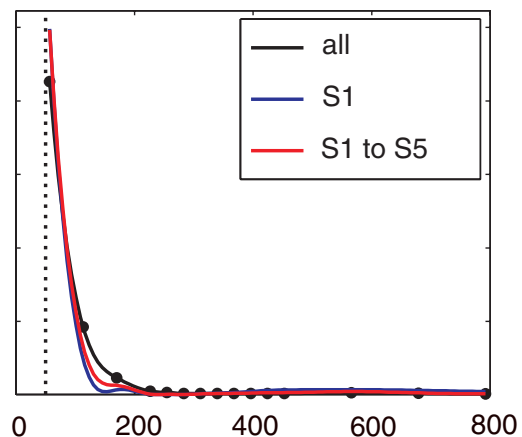

(c)

$P\left(\delta_{t} / \delta_{\mathrm{CWl}}=150 \mathrm{~m}\right)$

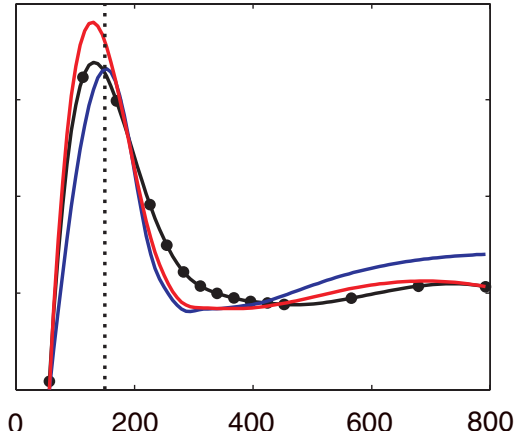

$(e)$

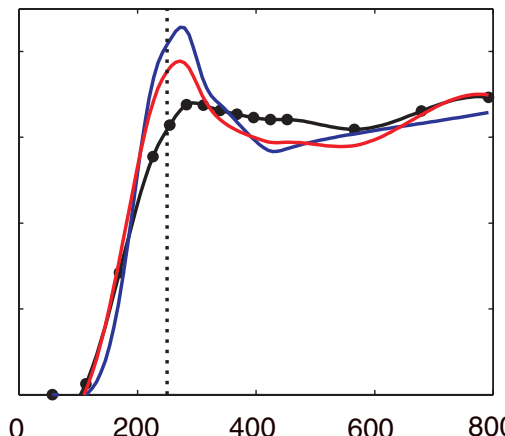

$(g)$

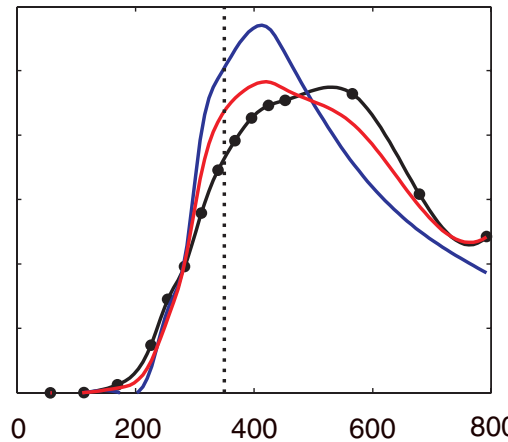

(b) $\quad P\left(\delta_{t} / \delta_{\mathrm{CWI}}=100 \mathrm{~m}\right)$

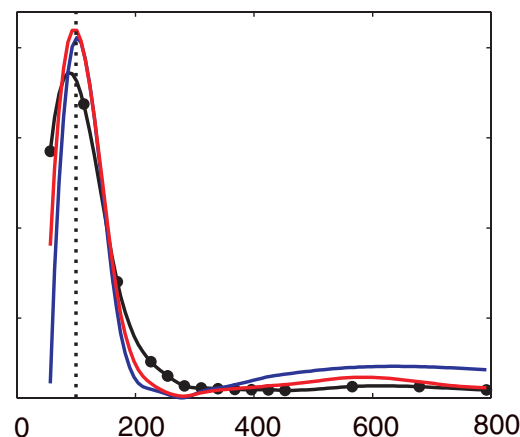

(d)

$P\left(\delta_{t} / \delta_{\mathrm{CWI}}=200 \mathrm{~m}\right)$

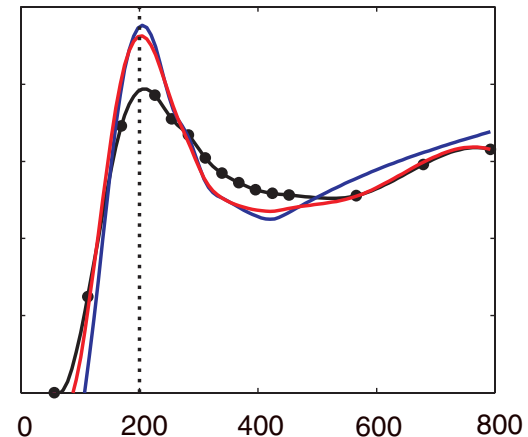

$(f)$

$P\left(\delta_{t} / \delta_{\mathrm{CWl}}=300 \mathrm{~m}\right)$

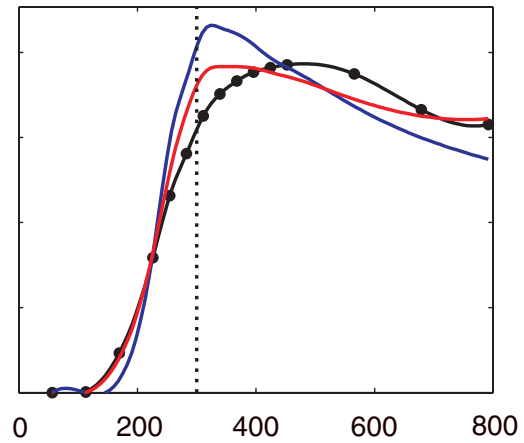

(h)

$P\left(\delta_{t} / \delta_{\mathrm{CWI}}=400 \mathrm{~m}\right)$

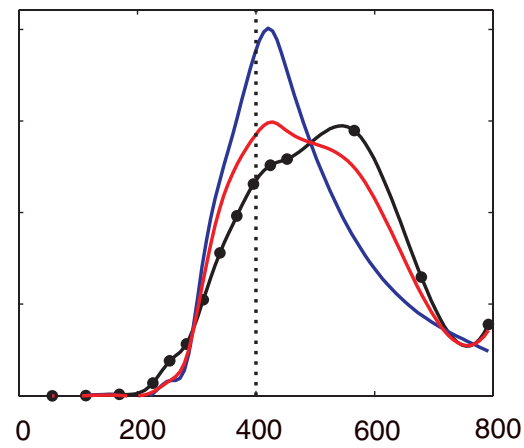

Fig. 9. Conditional probability density function $P\left(\delta_{t} \mid \delta_{\mathrm{CWI}}\right)$ for actual separation $\delta_{t}$ given CWI separation estimates $\delta_{\mathrm{CWI}}$ of (a) $50 \mathrm{~m},(b) 100 \mathrm{~m},(c) 150 \mathrm{~m},(d) 200 \mathrm{~m},(e) 250 \mathrm{~m},(f) 300 \mathrm{~m},(g)$ $350 \mathrm{~m}$ and $(h) 400 \mathrm{~m}$. Black, blue, and red curves represent $P\left(\delta_{t} \mid \delta_{\mathrm{CWI}}\right)$ when all stations, the single station $\mathrm{S} 1$ and stations $\mathrm{S} 1$ to $\mathrm{S} 5$ are used, respectively. The $y$-axis scale is omitted because each cross section of the conditional PDF is amplitude-normalised to emphasise its major features.

that the incorporation of CWI techniques will lead to improved accuracy when used in tandem with existing relative location techniques.
Potential applications of CWI to relative earthquake location problems include reconstruction of fault geometry by relocating aftershocks. Snieder and Vrijlandt (2005) demonstrated the 


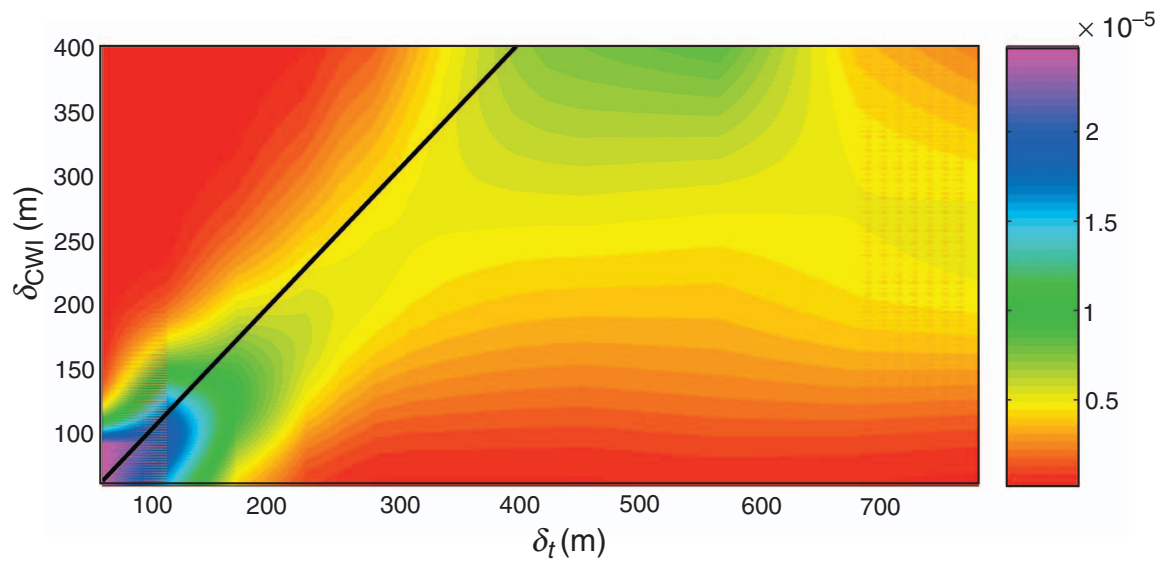

Fig. 10. Two-dimensional view of $P\left(\delta_{t} \mid \delta_{\mathrm{CWI}}\right)$.

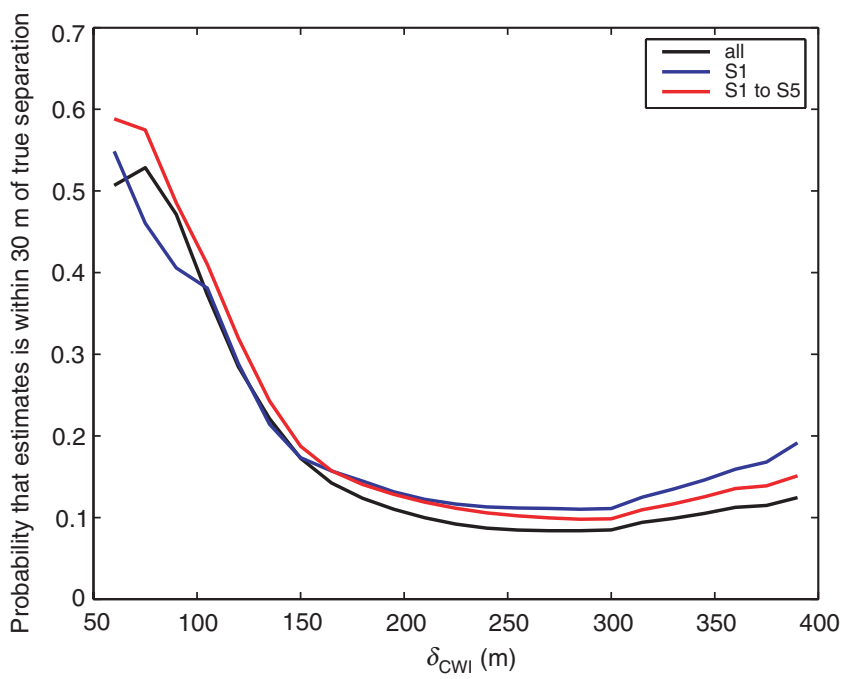

Fig. 11. Probability that CWI separation estimate is within $30 \mathrm{~m}$ of actual separation.

successful application of CWI for estimating the source separation between 3 pairs of events on the Hayward Fault, California. In this case, the CWI separation estimates (obtained from single stations) compared well with estimates from the double difference technique of Waldhauser and Ellsworth (2002) (which required the use of multiple stations). The event pairs considered had separations between 50 and $150 \mathrm{~m}$. Another potential application of CWI includes the analysis of microseimicity. It is common for many events to occur at small separations from one another in microseismic sequences. For example, an analysis of the 4357 events studied by Ake et al. (2005) Paradox Valley, Colorado reveals that all events occur in a volume $3(\mathrm{NS}) \times 6(\mathrm{EW}) \times 2($ depth $)$ $\mathrm{km}$ and that there are 370000 pair-wise separations less than $250 \mathrm{~m}$

We show that the PDF of interest $P\left(\delta_{t} \mid \delta_{\mathrm{CWI}}\right)$, the conditional probability of the true separation $\delta_{t}$ given the CWI separation estimate $\delta_{\mathrm{CWI}}$, is asymmetric. This implies that CWI estimates cannot be used with a quadratic penalty function as in a standard least-squares approach. Rather, the entire shape of the conditional PDF must be considered, something that can be achieved via maximum likelihood or Bayesian inversion algorithms (Mosegaard and Sambridge, 2002; Aster et al., 2005).

The velocity model used in the experiments has been chosen to produce a high level of scattering. Perturbations in the velocity model exceed that which is expected in the crust and the wave propagation is simplified by ignoring second order effects such as velocity gradients and layering. We show that even in such idealised conditions the CWI technique is limited to providing a lower bound for the true separation of events that are separated by more than $250 \mathrm{~m}$. Preliminary investigations in other random media suggest that the $250 \mathrm{~m}$ cutoff is controlled by the dominant frequency of the waveforms and not the perturbation size of the random medium. The dominant frequency of the waveforms will depend on the nature of the earthquake sources and the scattering properties of the medium. However, filtering can control it and there is no need to have prior knowledge of the velocity perturbations when applying CWI. Consequently, CWI can provide accurate estimates of separation at marginally greater distances by filtering the waveform to smaller frequencies (larger wavelengths).

The experiments provide no evidence to suggest that the free surface effect adversely influences CWI estimates. However, we detect a tendency for CWI to under-estimate the true separation as the source-to-station distance increases. Further work is required to quantify a relationship between CWI performance and the ratio $d / l_{s}$ where $d$ is the source-to-station distance and $l_{s}$ the mean free path of the medium.

\section{Conclusion}

We use numerical experiments to demonstrate that for waveforms filtered between 1 and $5 \mathrm{~Hz}$, coda wave interferometry is accurate for source separations of $250 \mathrm{~m}$ or smaller. Further work is required to quantify how the cut-off value of $250 \mathrm{~m}$ varies with frequency content in the waveforms and how the applicability of CWI depends on the source-to-station distance. Separation estimates from CWI are independent of arrival-time information and they do not require data from multiple stations. The synthetic experiment provides no evidence to suggest that the free surface has an adverse effect on the ability of CWI to estimate source separation.

We show how to compute the conditional probability density function (PDF) of actual separation given a CWI estimate. The computed conditional PDF provides a convenient mechanism for interpreting CWI separation and in addition, can be used as the basis for relative earthquake location.

\section{Acknowledgments}

This work has received the support of the Research School of Earth Sciences at The Australian National University; Geoscience Australia, an Australian Federal Government Agency; and the Center for Wave Phenomena at the Colorado School of Mines. Heiner Igel is thanked for supplying the original 
acoustic finite difference software. The manuscript has been improved courtesy of reviews by Marthijn de Kool (GA), Jürg Hauser (RSES), Matthew Purrs (GA), an anonymous reviewer, David Denham and the editorial team of Exploration Geophysics (Leonie Jones and Lindsay Thomas).

\section{References}

Ake, J., O'Connell, D., and Block, L., 2005, Deep-injection and closely monitored induced seismicity at Paradox Valley, Colorado: Bulletin of the Seismological Society of America 95, 664-683. doi: 10.1785/0120040072

Aster, R. C., Borchers, B., and Thurber, C. H., 2005, Parameter Estimation and Inverse Problems: Elsevier Academic Press.

Baig, A. M., and Dahlen, F. A., 2004, Statistics of traveltimes and amplitudes in random media: Geophysical Journal International 158, 187-210. doi: 10.1111/j.1365-246X.2004.02300.x

Bokelmann, G. H. R., and Harjes, H., 2000, Evidence for temporal variation of seismic velocity within the upper continental crust: Journal of Geophysical Research 105(B10), 23879-23894. doi: 10.1029/ 2000JB900207

Bondár, I., Myers, S. C., Engdahl, E. R., and Bergman, E. A., 2004, Epicentre accuracy based on seismic network criteria: Geophysical Journal International 156, 483-496. doi: 10.1111/j.1365-246X.2004.02070.x

Courant, R., Friedrichs, K., and Lewy, H., 1928, Über die partiellen Differenzengleichungen der mathematischen Physik: Mathematische Annalen 100, 32-74. doi: 10.1007/BF01448839

Deichmann, N., and Garcia-Fernandez, M., 1992, Rupture geometry from high precision relative hypocentre locations of microearthquake clusters: Geophysical Journal International 110, 501-517. doi: 10.1111/j.1365246X.1992.tb02088.x

De Rosny, J., and Roux, P., 2001, Multiple scattering in a reflective cavity: application to fish counting in a tank: The Journal of the Acoustical Society of America 109, 2587-2597. doi: 10.1121/1.1369101

Frankel, A., and Clayton, R. W., 1986, Finite difference simulations of seismic scattering: implications for the propagation of short-period seismic waves in the crust and models of crustal heterogeneity: Journal of Geophysical Research 91(B6), 6465-6489.

Frèmont, M.-J., and Malone, S. D., 1987, High precision relative locations of earthquakes at Mount St. Helens: Journal of Geophysical Research 92(B10), 10223-10236.

Got, J.-L., Frèchet, J., and Klein, F. W., 1994, Deep fault plane geometry inferred from multiplet relative relocation beneath the south flank of Kilauea: Journal of Geophysical Research 99(B8), 15375-15386. doi: 10.1029/94JB00577

Gutenberg, B., and Richter, C. F., 1939, New evidence for a change in physical conditions at depths near 100 kilometers: Bulletin of the Seismological Society of America 29, 531-537.

Ito, A., 1985, High resolution relative hypocenters of similar earthquakes by cross-spectral analysis method: Journal of Physics of the Earth 33, 279-294.

Lees, J. M., 1998, Multiplet analysis at Coso Geothermal: Bulletin of the Seismological Society of America 88, 1127-1143.

Mosegaard, K., and Sambridge, M., 2002, Monte Carlo analysis of inverse problems: Inverse Problems 18, R29-R54. doi: 10.1088/0266$5611 / 18 / 3 / 201$
Nadeau, R. M., and McEvilly, T. V., 1997, Seismological studies at Parkfield V: characteristic microearthquake sequences as fault-zone drilling targets: Bulletin of the Seismological Society of America 87, 1463-1472.

Pavlis, G. L., 1992, Appraising relative earthquake location errors: Bulletin of the Seismological Society of America 82, 836-859.

Poupinet, G., Ellsworth, W. L., and Frechet, J., 1984, Monitoring velocity variations in the crust using earthquake doublets: an application to the Calaveras Fault, California: Journal of Geophysical Research 89(B7), 5719-5731.

Richards, P. G., Waldhauser, F., Schaff, D., and Kim, W.-Y., 2006, The applicability of modern methods of earthquake location: Pure and Applied Geophysics 163, 351-372. doi: 10.1007/s00024-005-0019-5

Robinson, D. J., Snieder, R., and Sambridge, M., Using coda wave interferometry for estimating the variation in source mechanism between double couple events: Journal of Geophysical Research In press.

Rubin, A., 2002a, Using repeating earthquakes to correct highprecision earthquake catalogs for time-dependant station delays: Bulletin of the Seismological Society of America 92, 1647-1659. doi: 10.1785/0120010180

Rubin, A. M., 2002b, Aftershocks of microearthquakes as probes of the mechanics of rupture: Journal of Geophysical Research 107(B7), 2142. doi: 10.1029/2001JB000496

Rubin, A. M., Gillard, D., and Got, J.-L., 1999, Streaks of microearthquakes along creeping faults: Nature 400, 635-641. doi: 10.1038/23196

Scales, J. A., and Van Wijk, K., 2001, Tunable multiple-scattering system: Applied Physics Letters 79, 2294-2296 doi: 10.1063/1.1402156

Shearer, P., Hauksson, E., and Lin, G., 2005, Southern California hypocenter relocation with waveform cross-correlation, Part 2: Results using sourcespecific station terms and cluster analysis: Bulletin of the Seismological Society of America 95, 904-915. doi: 10.1785/0120040168

Shearer, P. M., 1999, Introduction to Seismology: Cambridge University Press.

Snieder, R., 1999, Imaging and averaging in complex media: in Fouque, J. P. (ed.). Diffuse waves in complex media, Volume 531 of NATO Science Series C, 405-454, Kluwer Academic Publishers

Snieder, R., 2004, Extracting the Green's function from the correlation of coda waves: a derivation based on stationary phase: Physical Review E: Statistical, Nonlinear, and Soft Matter Physics 69, 046610.

Snieder, R., 2006, The theory of coda wave interferometry: Pure and Applied Geophysics 163, 455-473. doi: 10.1007/s00024-005-0026-6

Snieder, R., and Vrijlandt, M., 2005, Constraining the source separation with coda wave interferometry: theory and application to earthquake doublets in the Hayward Fault, California: Journal of Geophysical Research 110(B04301), doi: 10.1029/2004JB003317

Waldhauser, F., and Ellsworth, W. L., 2002, Fault structure and mechanics of the Hayward Fault, California, from double-difference earthquake locations: Journal of Geophysical Research 107(B3), doi: 10.1029/2000JB000084

Waldhauser, F., Ellsworth, W. L., and Cole, A., 1999, Slip-parallel lineations on the Northern Hayward Fault, California: Geophysical Research Letters 26, 3525-3528. doi: 10.1029/1999GL010462

Manuscript received 4 August 2006; accepted 31 July 2007. 